\title{
A WIN-WIN STRATEGY FOR AN INTEGRATED VENDOR-BUYER DETERIORATING INVENTORY SYSTEM
}

\author{
P.C. YANG ${ }^{1}$ and H.M. WEE ${ }^{2}$ \\ ${ }^{1}$ Industrial Engineering and Management Department \\ St. John's \& St. Mary's Institute of Technology \\ Tamsui 25135 Taipei ROC \\ ${ }^{2}$ Industrial Engineering Department, Chung Yuan Christian University \\ Chungli 32023 Taiwan ROC \\ E-mail: pcyang@mail.sju.edu.tw; weehm@cycu.edu.tw
}

Received June 13, 2005; revised September 14, 2005

\begin{abstract}
An integrated approach considering the view of both the buyer and the vendor is discussed in this study. It can be shown numerically that the integrated approach results in an impressive cost reduction when compared with an independent decision approach by the buyer. Although the integrated total cost decreases, the buyer's cost increases due to larger orders. To entice the buyer to accept larger order quantity, a permissible delay in payment is offered by the vendor to the buyer. A negotiation factor is also incorporated to share the benefits.

Key words: integration, deterioration, permissible delay in payment, win-win strategy

\section{Introduction}

In general, a retailer has the privilege to decide on the lot size when an order is made. The optimal decision derived solely from the perspective of the buyer may not be the most economical one for the vendor. However, if both of their perspectives are taken into account, a joint policy can be achieved. The idea of vendor-buyer integration has been studied in the sixties by Clark and Scarf [2]. Ha and Kim [6] studied the integrated decision of the buyer and the vendor using JIT (just in time) purchasing. Banerjee [1] derived a joint economic lot size with finite production rate. Goyal [5] extended Banerjee's model by relaxing the lot-for-lot production assumption. Wee and Jong [16] considered the integration between multiple parts and finished product with multi-lot-size.
\end{abstract}


Deterioration is defined as decay, spoilage, evaporation, and loss of utility or loss of marginal value of a commodity that results in decreasing usefulness from the original one [15]. IC chip, blood, fish, strawberries, alcohol, gasoline, radioactive chemicals and grain products are examples of deteriorating commodities. Several researchers have studied deteriorating inventory in recent decades. Ghare and Schrader [4] were the first authors to consider on-going deterioration of inventory. Other authors such as Kang and Kim [8], Mak [11], Raafat et al. [13] and Heng et al. [7] assumed either instantaneous or finite production with different assumptions on the pattern of deterioration.

Kingsman [9] analyzed the relationship between the inventory cost and the payment rules. Davis and Gaither [3] derived the optimal ordering policies with extended payment. Mandal and Phaujdar [12] developed the EOQ model with permissible delay in payment. Liao et al. [10] derived an optimal replenishment time interval for deteriorating item with extended payment.

In this study, an integrated vendor-buyer inventory system for deteriorating item is developed. A negotiation factor is used to balance the benefit, and a permissible delay in payment is offered to the buyer to make the cooperation relationship more realistic and mutual beneficial.

\section{Mathematical Modeling and Analysis}

An inventory system of single-vendor and single-buyer with instantaneous replenishment is considered. If the deterioration rate is proportional to onhand stock, the inventory system at the vendor or the buyer with constant demand rate is represented by the following initial value differential problems:

$$
\left\{\begin{array}{l}
\frac{d I_{b}(t)}{d t}+\theta I_{b}(t)=-d, \quad 0 \leq t \leq T / n, \\
I_{b}(t)=0, \quad t=T / n
\end{array}\right.
$$

and

$$
\left\{\begin{array}{l}
\frac{d I_{\nu}(t)}{d t}+\theta I_{\nu}(t)=-d, \quad 0 \leq t \leq T \\
I_{\nu}(t)=0, \quad t=T .
\end{array}\right.
$$

The solutions of this differential problems are [14]:

$$
\begin{aligned}
& I_{b}(t)=\frac{d}{\theta}(\exp (\theta(T / n-t))-1), \quad 0 \leq t \leq T / n, \\
& I_{\nu}(t)=\frac{d}{\theta}(\exp (\theta(T-t))-1), \quad 0 \leq t \leq T .
\end{aligned}
$$

The order quantities for the buyer and the vendor are $I_{b}(0)$ and $I_{\nu}(0)$ respectively, and they are defined as follows:

$$
I_{m b}=\frac{d}{\theta}(\exp (\theta T / n)-1), \quad I_{m \nu}=\frac{d}{\theta}(\exp (\theta T)-1) .
$$


In the time period $T$, the total holding cost for the buyer is

$$
\text { Holding cost of the buyer }=C_{b 2} n \int_{0}^{T / n} I_{b}(t) d t .
$$

The actual vendor average inventory level in the integrated two-echelon inventory model is the difference between the vendor-buyer combined average inventory level and the buyer average inventory level. The actual vendor holding cost in time $T$ is expressed as:

$$
\text { Holding cost of the vendor }=C_{\nu 2}\left[\int_{0}^{T} I_{\nu}(t) d t-n \int_{0}^{T / n} I_{b}(t) d t\right] .
$$

In the time period $T$,

$$
\text { the deterioration cost for the buyer }=C_{b} n\left[I_{m b}-\frac{d T}{n}\right]
$$

and

$$
\text { the deterioration cost for the vendor }=C_{b} n\left[I_{m \nu}-I_{m b}\right] \text {. }
$$

In the time period $T$,

$$
\text { the ordering costs for the buyer }=C_{b 1} n
$$

and

$$
\text { the ordering costs for the vendor }=C_{\nu 1} \text {. }
$$

The annual buyer's total cost, $T C_{b}$ is the sum of (2.3), (2.5) and (2.7) divided by $T$ :

$$
T C_{b}=\frac{C_{b 2} n}{T} \int_{0}^{\frac{T}{n}} I_{b}(t) d t+\frac{C_{b} n}{T}\left[I_{m b}-\frac{d T}{n}\right]+\frac{C_{b l} n}{T} .
$$

The annual vendor's total cost $T C_{\nu}$ is the sum (2.4), (2.6) and (2.8) divided by $T$ :

$$
T C_{\nu}=\frac{C_{\nu 2} n}{T}\left[\int_{0}^{T} I_{\nu}(t) d t-n \int_{0}^{\frac{T}{n}} I_{b}(t) d t\right]+\frac{C_{\nu}}{T}\left[I_{m \nu}-n I_{m b}\right]+\frac{C_{\nu 1}}{T} .
$$

The annual integrated total cost $T C$ is the sum of $T C_{b}$ and $T C_{v}$. Since $T=$ $n T_{b}, T C$ is a function of two variables, $T_{b}$ and $n$.

$$
T C=T C_{b}+T C_{\nu} .
$$




\section{Solution Procedure}

For the case without considering integration, the buyer and the vendor make strategic decision independently. In buyer market, the first step is for the buyer to minimize $T C_{b}$ with respect to $T_{b}$ as follow:

$$
\frac{\partial T C_{b}}{\partial T_{b}}=0 .
$$

The point satisfying (3.1) is the minimum of the buyer's total cost (see Appendix A). The second step is for the vendor to minimize $T C_{v}$ by deriving the replenishment times per cycle time. Since the replenishment time is a discrete integer, it must satisfy the following condition:

$$
T C_{\nu}(n-1) \geq T C_{\nu}(n) \leq T C(n+1) .
$$

The total cost without considering integration, $T C^{\#}$ is expressed as

$$
T C^{\#}=\min _{n}\left[\left(\min _{T_{n}} T C_{b}\right)+T C_{\nu}\right] .
$$

For the case considering integration, the total cost is optimized jointly rather than independently. For a fixed $n, T_{b}$ is derived from (3.3) and denoted by $T_{b}(n)$. The optimal values of $T_{b}$ and $n$, denoted by $T_{b}^{*}\left(n^{*}\right)$ and $n^{*}$, must satisfy the following conditions simultaneously:

$$
\begin{gathered}
\frac{\partial T C}{\partial T_{b}}=0 \\
T C\left(T_{b}\left(n^{*}-1\right), n^{*}-1\right) \geq T C\left(T_{b}^{*}\left(n^{*}\right), n^{*}\right) \leq T C\left(T_{b}\left(n^{*}+1\right), n^{*}+1\right) .
\end{gathered}
$$

$T C$ is convex with respect to $T_{b}$ (see Appendix B).

The total cost considering integration, $T C^{*}$ is expressed as

$$
T C^{*}=\min _{T_{b}, n}\left(T C_{b}+T C_{\nu}\right) .
$$

The point satisfying (3.3) and (3.5) is the minimum of the integrated total cost (see Appendix C).

Since (3.5) is less than (3.2), the total cost saving, $S_{\text {int }}$ is defined as

$$
S_{\text {int }}=T C^{\#}-T C^{*} .
$$

Let the buyer's cost saving, $S_{b}$ be defined as

$$
S_{b}=\alpha S_{i n t},
$$

where $\alpha$ is the negotiation factor. When the negotiation factor equals one, it means all saving is accrued to the buyer; when the negotiation factor is 0.5 , it implies that the total cost saving is equally distributed between the 
vendor and the buyer. If the negotiation factor is zero, all saving is given to the vendor.

A permissible delay in payment is used to entice the buyer to cooperate in the integrated inventory system. The present value of unit cost after a time interval $\Delta t$ is $e^{-R \Delta t}$. The buyer's permissible delay in payment can be derived solving the following equation:

$$
d C_{b}\left(1-e^{-R \Delta t}\right)=S_{b},
$$

where $R$ is the continuous interest rate. The close form solution for $\Delta t$ is

$$
\Delta t=\frac{1}{R} \ln \left[\frac{C_{b} d}{C_{b} d-\alpha\left(T C^{\#}-T C^{*}\right)}\right] .
$$

\section{Numerical Example}

The preceding theory can be illustrated by the following numerical example. The annual demand is 40,000 units. The unit costs for the vendor and the buyer are $\$ 10$ and $\$ 12$ respectively. The ordering cost for the vendor and the buyer are $\$ 3,000$ and $\$ 600$ respectively. The annual percentage of holding cost for the vendor and the buyer are $10 \%$ and $11 \%$ per unit. The annual deterioration rate is $10 \%$. The interest rate and the negotiation factor are assumed to be $3 \%$ and 0.5 respectively.

By applying the solution procedure from Section 3, the solution comparing the case with integration to the case without integration is given in Table 1. The buyer's cost and the replenishment interval increase, when integration is considered. The vendor benefits $\$ 6,698$, while the buyer losses $\$ 4,717$. Therefore, the buyer will be hesitant to support the integration process. To entice the buyer to cooperate, the vendor offers the buyer a permissible delay in payment of 0.06887 years with equally distributed benefit. The percentage of integrated total cost reduction $(P I C R)$ is defined as PICR $=\frac{T C^{\#}-T C^{*}}{T C^{\#}}$. The value of $P I C R$ is $6.82 \%$.

Table 1. The optimal solution with and without considering integration. Note: $P I C R=\left(T C^{\#}-T C^{*}\right) / T C^{\#}$.

\begin{tabular}{lcc}
\hline Cases & Without integration & With integration \\
\hline$n$ & 3 & 1 \\
$T_{b}$ & 0.1087 & 0.2649 \\
$T$ & 0.3261 & 0.2649 \\
$T C_{b}$ & $\$ 11,018$ & $\$ 15,735$ \\
$T C_{\nu}$ & $\$ 18,023$ & $\$ 11,325$ \\
$T C$ & $\$ 29,041$ & $\$ 27,060$ \\
$P I C R$ & - & $6.82 \%$ \\
$\Delta t$ (years) & - & 0.06887 \\
\hline
\end{tabular}




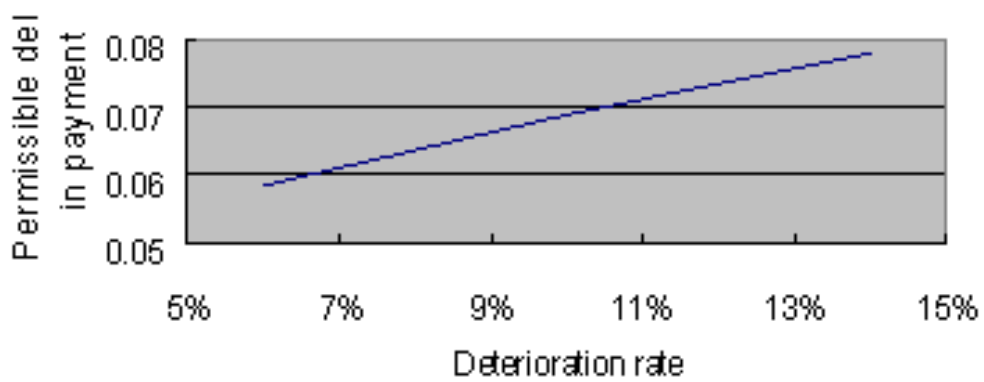

Figure 1. Permissible delay in payment vs. deterioration rate.

\section{Sensitivity Analysis}

Sensitivity analysis is carried out when one of the parameters is increased or decreased by $20 \%$ and $40 \%$ while the other parameters remain unchanged. The results are given in Tables $2-7$ and Figure 1 .

When the deterioration rates increase or the demand rates decrease, the PICR and the permissible delay in payment increase (Table 2 and Table 3 ). Therefore, the greater the deterioration rate or the less the demand rate, there is greater need to consider the integration process.

Table 2. Sensitivity analysis of deterioration rate. Note: \{\} is a base column.

\begin{tabular}{lccccc}
\hline$\theta$ & 0.06 & 0.08 & $\{0.10\}$ & 0.12 & 0.14 \\
$N^{\#}$ & 3 & 3 & 3 & 3 & 3 \\
$T C^{\#}$ & 25,991 & 27,557 & 29,041 & 30,455 & 31,810 \\
$n^{*}$ & 1 & 1 & 1 & 1 & 1 \\
$T C^{*}$ & 24,311 & 25,721 & 27,060 & 28,337 & 29,561 \\
$P I C R$ & $6,46 \%$ & $6,66 \%$ & $6,82 \%$ & $6,95 \%$ & $7,07 \%$ \\
$\Delta t$ & 0,05839 & 0,06382 & 0,06887 & 0,07361 & 0,07817 \\
\hline
\end{tabular}

From Table $4-5$, we can see that PICR and the permissible delay in payment are more sensitive to the buyer's ordering cost, and less sensitive to the vendor's ordering cost. From Table 6, the permissible delay in payment increases when the buyer's holding cost increases. Table 7 demonstrates that the negotiator factor does not influence PICR values, but influences significantly the permissible delay in payment.

When integration is considered, the percentage extra benefit (PEB) considering deterioration is defined as:

$$
P E B=\frac{T C^{*}-T C^{*}}{T C^{2}}
$$

where^represents the optimal value without considering deterioration. 
Table 3. Sensitivity analysis of demand rate.

\begin{tabular}{lccccc}
\hline$d$ & 24.000 & 32.000 & $\{40.000\}$ & 48.000 & 56.000 \\
$n^{\#}$ & 3 & 3 & 3 & 3 & 3 \\
$T C^{\#}$ & 22,529 & 25,991 & 29,041 & 31,799 & 34,334 \\
$n^{*}$ & 1 & 1 & 1 & 1 & 1 \\
$T C^{*}$ & 20,987 & 24,216 & 27,060 & 29,631 & 31,995 \\
$P I C R$ & $6,84 \%$ & $6,83 \%$ & $6,82 \%$ & $6,82 \%$ & $8,81 \%$ \\
$\Delta t$ & 0,08934 & 0,07715 & 0,06887 & 0,06279 & 0,05805 \\
\hline
\end{tabular}

Table 4. Sensitivity analysis of buyer's ordering cost.

\begin{tabular}{lccccc}
\hline$C_{b 1}$ & 360 & 480 & $\{600\}$ & 720 & 840 \\
$n^{\#}$ & 3 & 3 & 3 & 2 & 2 \\
$T C^{\#}$ & 27,215 & 28,017 & 29,041 & 29,491 & 29,919 \\
$n^{*}$ & 1 & 1 & 1 & 1 & 1 \\
$T C^{*}$ & 26,138 & 26,603 & 27,060 & 27,509 & 27,951 \\
$P I C R$ & $3,96 \%$ & $5,05 \%$ & $6,82 \%$ & $6,72 \%$ & $6,58 \%$ \\
$\Delta t$ & 0,03741 & 0,04914 & 0,06887 & 0,06889 & 0,06840 \\
\hline
\end{tabular}

Table 5. Sensitivity analysis of vendor's ordering cost.

\begin{tabular}{lccccc}
\hline$C_{v 1}$ & 1800 & 2400 & $\{3000\}$ & 3600 & 4200 \\
$n^{\#}$ & 2 & 2 & 3 & 3 & 3 \\
$T C^{\#}$ & 23,693 & 26,452 & 29,041 & 30,881 & 32,720 \\
$n^{*}$ & 1 & 1 & 1 & 1 & 1 \\
$T C^{*}$ & 22,076 & 24,692 & 27,060 & 29,238 & 31,267 \\
$P I C R$ & $6,82 \%$ & $6,65 \%$ & $6,82 \%$ & $5,32 \%$ & $4,44 \%$ \\
$\Delta t$ & 0,05618 & 0,06115 & 0,06887 & 0,05709 & 0,05048 \\
\hline
\end{tabular}

Table 6. Sensitivity analysis of buyer's ordering cost.

\begin{tabular}{lccccc}
\hline$C_{b 2}$ & 0,066 & 0,088 & $\{0,11\}$ & 0,132 & 0,154 \\
$n^{\#}$ & 3 & 3 & 3 & 3 & 3 \\
$T C^{\#}$ & 25,931 & 27,530 & 29,041 & 30,477 & 31,848 \\
$n^{*}$ & 1 & 1 & 1 & 1 & 1 \\
$T C^{*}$ & 24,072 & 25,609 & 27,060 & 28,436 & 29,748 \\
$P I C R$ & $7,17 \%$ & $6,98 \%$ & $6,82 \%$ & $6,70 \%$ & $6,59 \%$ \\
$\Delta t$ & 0,06462 & 0,06675 & 0,06887 & 0,07095 & 0,07300 \\
\hline
\end{tabular}

Table 7. Sensitivity analysis of negotiation factor.

\begin{tabular}{lccccc}
\hline$\alpha$ & 0.3 & 0.4 & $\{0.5\}$ & 0.6 & 0.7 \\
$n^{\#}$ & 3 & 3 & 3 & 3 & 3 \\
$T C^{\#}$ & 29,041 & 29,041 & 29,041 & 29,041 & 29,041 \\
$n^{*}$ & 1 & 1 & 1 & 1 & 1 \\
$T C^{*}$ & 27,060 & 27,060 & 27,060 & 27,060 & 27,060 \\
$P I C R$ & $6,82 \%$ & $6,82 \%$ & $6,82 \%$ & $6,82 \%$ & $6,82 \%$ \\
$\Delta t$ & 0,04130 & 0,05508 & 0,06887 & 0,08266 & 0,09645 \\
\hline
\end{tabular}


Table 8. Percentage extra benefit (PEB) when deterioration is considered.

\begin{tabular}{|c|c|c|c|c|c|c|}
\hline$\theta$ & 0.001 & 0.06 & 0.08 & $\{0.10\}$ & 0.12 & 0.14 \\
\hline$T_{b}$, & 36756 , & 10.36756 , & 10.36756 , & 10.36756 , & 10.36756 , & 10.36756 , \\
\hline$T C^{n}$ & 19,615 & 24,902 & 26,721 & 28,548 & 30,385 & 32,231 \\
\hline$n^{*}$ & 1 & 1 & 1 & 1 & 1 & 1 \\
\hline$T C^{*}$ & 19,61 & 24,3 & 25,72 & 27, & 28,337 & 29,561 \\
\hline$P E B$ & $0,00 \%$ & $2,37 \%$ & $3,74 \%$ & $\%$ & $6,74 \%$ & $8.28 \%$ \\
\hline$\Delta t$ & 0 & 0,05839 & 0,06382 & 0,06887 & 0,07361 & 0,07817 \\
\hline
\end{tabular}

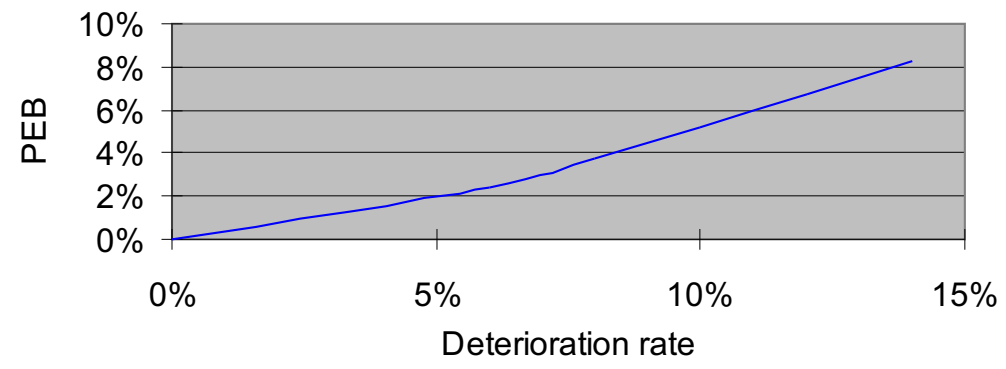

Figure 2. Percentage extra benefit vs. deterioration rate.

In the case without considering deterioration $(\theta=0.001 \approx 0)$, the optimal buyer's replenishment interval and replenishment times per cycle are 0.36756 years and one respectively. When the deterioration rates increase, the percentage extra benefits increase up to $8.28 \%$ (Table 8 and Figure 2). The computational result shows that it is significant to consider the deterioration factor in the supply chain integration.

\section{Conclusions}

In this paper, we develop a mathematical model for deteriorating item to derive an optimal ordering policy in the integrated vendor-buyer inventory system. It is shown that the optimal policy using the integrated approach has resulted in a lower joint cost. However, the buyer's cost increases when the integrated approach is used. To motivate the buyer's cooperation, an incentive system in the form of credit term to the buyer is incorporated into the system. In this analysis, we show that it is more significant to consider the integration process and the permissible delay in payment when deterioration rate and the holding rate are higher. 


\section{Appendix}

\section{A Proof of Convex of the Buyer's Total Cost}

The proof is given for the case when deterioration is considered. For $\theta T_{b} \ll 1$, $\exp \left(\theta T_{b}\right)$ is replaced by Taylor series approximation:

$$
1+\theta T_{b}+\left(\theta T_{b}\right)^{2} / 2 !+\left(\theta T_{b}\right)^{3} / 3 !
$$

The percentage error for the fourth term in Taylor series is

$$
\left[\left(\theta T_{b}\right)^{3} / 3 !\right] /\left[1+\theta T_{b}+\left(\theta T_{b}\right)^{2} / 2 !+\left(\theta T_{b}\right)^{3} / 3 !\right] .
$$

When $\theta=0.1$ and $T_{b}=0.2649$, the percentage error is about $0.0003017 \%$. It will be smaller for terms higher than fourth. The integrated total cost of (2.9) can be expressed as

$$
T C_{b}=\frac{1}{2 T_{b}}\left(C_{b 2} d T_{b}^{2}+C_{b} d T_{b}^{2} \theta+2 C_{b 1}\right) .
$$

The second derivatives of (A.1) with respect to $T_{b}$ is

$$
\frac{d^{2} T C_{b}}{d T_{b}^{2}}=\frac{2 C_{b 1}}{T_{b}^{3}}>0
$$

Since (A.2) is positive, the buyer's total cost is convex with respect to $T_{b}$.

\section{B Proof of Convex of the Integrated Total Cost with Respect to $T_{b}$}

The integrated total cost of (2.11) can be expressed as

$T C=\frac{1}{2 n T_{b}}\left[n d T_{b}^{2}\left(C_{b 2}+C_{b} \theta\right)+2\left(n C_{b 1}+C_{v 1}\right)+\left(C_{v 2}+C_{\nu} \theta\right) n d T_{b}^{2}(n-1)\right]$.

The second derivative of the integrated total cost with respect to $T_{b}$ is given as follow:

$$
\frac{d^{2} T C}{d T_{b}^{2}}=\frac{2\left(n C_{b 1}+C_{v 1}\right)}{n T_{b}^{3}}>0
$$

Since (B.2) is positive, $T C$ is convex with respect to $T_{b}$.

\section{Proof of the Minimum of the Integrated Total Cost with Respect to $n$ and $T_{b}$}

The integrated total cost of (2.11) can be expressed as 
$T C=\frac{1}{2 n T_{b}}\left[\left(C_{\nu 2}+C_{\nu} \theta\right) d T_{b}^{2} n(n-1)+2\left(C_{\nu 1}+n C_{b 1}\right)+d T_{b}^{2} n\left(C_{b 2}+C_{b} \theta\right)\right]$.

Since the downstream cost is larger than the upstream cost, $C_{b}$ and $C_{b 2}$ are larger than $C_{\nu}$ and $C_{\nu 2}$ respectively. One can assume:

$$
C_{b}=C_{\nu}+r, \quad C_{b 2}=C_{\nu 2}+s,
$$

where $r$ and $s$ are all positive real values. Equating the first derivatives of $T C$ with respect to $n$ to zero, the unique feasible value of $n$ is solved as:

$$
n=\frac{1}{T_{b}} \sqrt{\frac{2 C_{\nu 1}}{d\left(C_{\nu 2}+C_{\nu} \theta\right)}} .
$$

Substituting (C.3) into (C.1), the integrated total cost can be expressed as function of only one variable, buyer's cycle time $T_{b}$. Equating the first derive of $T C$ with respect to buyer's cycle time to zero, the cycle time can be solved as follow:

$$
T_{b}=\sqrt{\frac{2 C_{b 1}}{(s+r \theta)} d}
$$

The sufficient conditions for the extreme points of the integrated total cost are $\frac{\partial T C}{\partial n}=0$ and $\frac{\partial T C}{\partial T_{b}}=0$. There are four extreme points: one is feasible, the other three are non-feasible. These are:

$$
\begin{aligned}
& \left(n, T_{b}\right)=\left(\sqrt{\frac{C_{\nu 1}(s+r \theta)}{C_{b 1}\left(C_{\nu 2}+C_{\nu} \theta\right)}}, \sqrt{\frac{2 C_{b 1}}{(s+r \theta)}} d\right), \quad \text { (feasible) } \\
& \left(n, T_{b}\right)=\left(-\sqrt{\frac{C_{\nu 1}(s+r \theta)}{C_{b 1}\left(C_{\nu 2}+C_{\nu} \theta\right)}}, \sqrt{\frac{2 C_{b 1}}{(s+r \theta)} d}\right), \quad \text { (non-feasible) } \\
& \left.\left(n, T_{b}\right)=\left(\sqrt{\frac{C_{\nu 1}(s+r \theta)}{C_{b 1}\left(C_{\nu 2}+C_{\nu} \theta\right)}},-\sqrt{\frac{2 C_{b 1}}{(s+r \theta)} d}\right), \quad \text { (non }- \text { feasible }\right) \\
& \left(n, T_{b}\right)=\left(-\sqrt{\frac{C_{\nu 1}(s+r \theta)}{C_{b 1}\left(C_{\nu 2}+C_{\nu} \theta\right)}},-\sqrt{\frac{2 C_{b 1}}{(s+r \theta)} d}\right), \quad(\text { non }- \text { feasible })
\end{aligned}
$$

After substituting (C.3) into (C.1), the second derivative of $T C$ with respect to buyer's cycle time is

$$
\frac{d^{2} T C}{d T_{b}^{2}}=\frac{2 C_{b 1}}{T_{b}^{3}}>0
$$

If $n$ and $T_{b}$ are any real numbers, the point of (C.5) is the minimum in the feasible area. When $n$ is fixed, $T_{b}$ is derived from (C.11) and denoted by $T_{b}(n)$. If $n$ is integral and $T_{b}$ is any real number, the optimal point, denoted by $n^{*}$ and $T_{b}^{*}\left(n^{*}\right)$, satisfying the following conditions simultaneously is the minimum. 


$$
T C\left(n^{*}-1, T_{b}\left(n^{*}-1\right)\right) \geq T C\left(n^{*}, T_{b}^{*}\left(n^{*}\right)\right) \leq T C\left(n^{*}+1, T_{b}\left(n^{*}+1\right)\right)
$$

and

$$
\frac{d T C}{d T_{b}}=0 \quad \forall n
$$

\section{Nomenclature}

$\theta \quad$ Deterioration rate per year

$I_{m v}$ Order quantity for the vendor

$I_{m b}$ Order quantity for the buyer to the vendor

$T \quad$ Vendor's replenishment time interval (year)

$T_{b} \quad$ Buyer's replenishmenttime interval (year)

$n$ Buyer's order timesperT

$d \quad$ Annual demand rate

$C_{v} \quad$ Vendor's unit cost

$C_{b} \quad$ Buyer's unit cost

$I_{v}(t)$ Vendor - buyer combined inventory level

$I_{b}(t)$ Buyer's inventory level

$C_{v 1}$ Vendor's ordering cost per setup

$C_{b 1}$ Buyer's ordering cost per setup

$C_{v 2}$ Vendor's annual holding cost per unit

$C_{b 2}$ Buyer's annual holding cost per unit

$T C_{v}$ Vendor's annual total cost

$T C_{b}$ Buyer's annual total cost

$T C$ Annual total cost for both vendor and buyer

$\Delta t \quad$ Permissible delay in payment offered by the vendor to the buyer (year)

$R \quad$ Continuous interest rate per year

$\alpha \quad$ Negotiation factor

\section{References}

[1] A. Banerjee. A joint economic lot size model for purchaser and vendor. Decision Sciences, 17, $292-311,1986$.

[2] A.J. Clark and H. Scarf. Optimal policies for a multi-echelon inventory problem. Management Science, 6, 475 - 490, 1960.

[3] R.A. Davis and N. Gaither. Optimal ordering policies under conditions of extended payment privileges. Management Science, 31, 499 - 509, 1985.

[4] P.M. Ghare and S.F. Schrader. A model for exponentially decaying inventory. Journal of Industrial Engineering, 14(5), 238 - 243, 1963.

[5] S.K. Goyal. A joint economic lot size model for purchaser and vendor: A Comment. Decision Sciences, 19, 236 - 241, 1988.

[6] D. Ha and S.L. Kim. Implementation of JIT purchasing:an integrated approach. Production Planning \& Control, 8(2), 152 - 157, 1997. 
[7] K.J. Heng, J. Labban and R.L. Lim. An order-level lot size inventory model for deteriorating items with finite replenishment rate. Computers $\&$ Industrial Engineering, 20, 187 - 197, 1991.

[8] S. Kang and I. Kim. A study on the price and production level of the deteriorating inventory system. International Journal of production Research, 21, 449 $-460,1983$.

[9] B.G. Kingsman. The effect of the payment rules on ordering and stocking in purchasing. Journal of the Operational Research Society, 48, 1085 - 1098, 1997.

[10] H.C. Liao, C.H. Tsai and C.T. Su. An inventory model with deteriorating items under inflation when a delay in payment is permissible. International Journal of Production Economics, 63, 207 - 214, 2000.

[11] K.L. Mak. A production lot size inventory model for deteriorating items. Computers 6 Industrial Engineering, 6, 309 - 317, 1982.

[12] B.N. Mandal and S. Phaujdar. Some EOQ models under permissible delay in payments. International Journal of Managements Science, 5(2), 99 - 108, 1989.

[13] F. Raafat, P.M. Wolfe and H.K. Eldin. An inventory model for deteriorating items. Computers \& Industrial Engineering, 20, 89 - 94, 1991.

[14] M.R. Spiegel. Applied Differential Equations. Prentice-Hall,Eagle wood Cliffs, NJ, 1960.

[15] H.M. Wee. Economic production lot size model for deteriorating items with partial backordering. Computers \& Industrial Engineering, 24(3), 449 - 458, 1993.

[16] H.M. Wee and J.F. Jong. An integrated multi-lot-size production inventory model for deteriorated items. Management \& System, 5(1), 97 - 114, 1998. 